Research Article

\title{
Design and Implementation of Intelligent Educational Administration System Using Fuzzy Clustering Algorithm
}

\author{
Fang Liu (iD \\ Scientific Research Department, Xijing University, Xi'an, Shaanxi 710123, China \\ Correspondence should be addressed to Fang Liu; sunrain110312@163.com
}

Received 24 October 2021; Revised 17 November 2021; Accepted 25 November 2021; Published 29 December 2021

Academic Editor: Le Sun

Copyright (C) 2021 Fang Liu. This is an open access article distributed under the Creative Commons Attribution License, which permits unrestricted use, distribution, and reproduction in any medium, provided the original work is properly cited.

\begin{abstract}
The present work aims to solve the problems that the traditional educational administration management system has, such as low efficiency in analyzing big data, and the analysis results have low value, which is based on manual rules definition in big data analysis and processing. The work proposes a student achievement prediction model FCM-CF based on Fuzzy C-means (FCM) and Collaborative Filtering (CF). The work also introduces it into the research of educational administration management to construct an intelligent educational administration management system. At the beginning, the FCM-CF model is described in detail. Then, the system requirements and specific design methods are described in detail. Eventually, with the students' performance prediction as an example, the performance of the system is tested by designed simulation experiments. The result shows that the students' achievement in study is closely related to their daily study performance such as preparation before class, classroom performance, attendance, extracurricular study, and homework completion. Generally, the examination scores of students are significant to their daily performances. Under the same experimental conditions, the prediction error of the FCM-CF model proposed here is less than $10.8 \%$ of that of other algorithms. The model has better prediction performance and is more suitable for the prediction of middle school students' examination scores in educational administration management system. The innovation of intelligent educational administration management system is that, in addition to the basic information management function, it also has two other functions: students' performance prediction analysis and teacher evaluation prediction. It can provide data support for improving teaching quality. The research purpose is to provide important technical support for more intelligent educational administration and reduce the loss of human resources in educational administration.
\end{abstract}

\section{Introduction}

In recent years, with the continuous development of computer technology, people's life has entered a highly information-based era. Whether it is personal identity information, consumption information, or travel information, while bringing great convenience to people, they have been familiar by people. Of course, this convenience is also reflected in the education industry, especially in colleges. The use of information technology has been very mature. In daily teaching, the application of computer-aided instruction (CAI) breaks the rigid traditional teaching mode and makes the lecture vivid and flexible [1]. In the aspect of campus management, the application of information technology makes the management of colleges become intelligent, which greatly reduces the waste of related manpower. The educational administration management system in colleges is the embodiment of this utilization. As the center of college management, educational administration management system manages a series of actions and plans of the college, including the students' status information, students' online course selection, teachers' annual teaching plan, teaching materials, ability test of each semester, statistical entry and query of test results, and management of teachers' evaluation of teaching [2].

Nasir et al. investigated the influencing factors of selfregulated academic performance of students majoring in management in the College of Teacher Training and Science Education. The results showed that students with higher academic performance would have stronger self-regulation ability [3]. Lv et al. designed the scheme of intelligent building indoor environment measurement, and the control 
system based on cyber-physical systems (CPS) included four basic modules: detection, control, execution, and communication. CPS and artificial intelligence (AI) were studied to promote their application in the construction industry. It is believed that the research on the combination of CPS and AI in the construction industry can provide theoretical basis for the development of intelligent building industry [4]. Xie et al. claimed that deep learning (DL) had shown great potential in disease prediction and drug response prediction, summarized the DL prediction methods for different diseases, pointed out a series of problems in current disease prediction, and proved the high correlation between DL and the future development of medical field [5]. Lv et al. built a smart city constructing model with the support of Internet of things, cloud computing, and Internet and stimulated the vertical market system of smart city from an economic perspective. The results suggested that the system had good stability. From the perspective of vertical market, selfoperated retailers have more advantages, which can provide experimental reference for future smart city construction and economic development [6].

With the expansion of scales of colleges and enrolment, the number of students and teachers is increasing. The large quantity of personnel information data brings difficulties to school management. However, most colleges and universities do not pay attention to the innovation of educational administration management. Their management systems are still designed by technical personnel directly through the definition of rules according to the actual situation and needs of the school. These systems can provide information query, adding and deleting functions, but cannot be used to analyze data. Therefore, the school enrolment plan analysis, teacher personnel management, teaching management, and asset equipment management need to be carried out by manual analysis and processing, and the efficiency is very low. Based on this, the present work proposes a student achievement prediction model-FCM-CF based on Fuzzy $\mathrm{C}$-means (FCM) and Collaborative Filtering (CF) and introduces it into the research of educational administration management to construct an intelligent educational administration management system. Primarily, the FCM-CF model is described in detail. Then, the system requirements and specific design methods are illustrated. Ultimately, taking the student performance prediction as an example, the performance of the system is tested by designed simulation experiments. The powerful data mining ability of FCM can also make colleges better deal with the real-time situation of education and make a certain contribution to the development and optimization of educational administration in colleges. The research purpose is to provide important technical support for more intelligent educational administration and reduce the loss of human resources in educational administration.

\section{Model Construction and System Design}

2.1. Students' Scores Prediction Model Based on FCM-CF. Clustering analysis is the process of using mathematical methods to study and process the given objects, and distinguishing and classifying them according to the similarity between things. With the establishment of fuzzy theory, people begin to use fuzzy methods to deal with clustering problems, namely, the fuzzy clustering analysis. Fuzzy clustering can be applied to deal with many objects whose attribute distinction is not obvious in practice. It uses membership function to express the similarity between samples. Since fuzzy clustering obtains the uncertainty degrees of number of samples and each category, it expresses the intermediary of the sample category; that is, it establishes the description of the uncertainty of the sample for the category, which can more objectively reflect the real world. Generally, the fuzzy clustering method based on objective function is used to cluster the targets [7]. Equation (1) represents the sample set $X=\left\{x_{1}, x_{2}, \ldots, x_{n}\right\}$.

$$
\sum_{j=1}^{c} \mu_{j k}=1 \text {. }
$$

In equation (1), $j=1,2, \ldots, c, k=1,2, \ldots, n$. Parameter $c(2 \leq c \leq n)$ is the number of categories of sample $X$ transferred. Parameter $\mu_{j k} \in[0,1]$ means the membership degree of sample $k$ to the class $j$.

The fuzzy partition matrix $U$ is defined as

$$
U=\left\{\begin{array}{c}
\mu_{11} \mu_{12} \ldots \mu_{1 n} \\
\mu_{21} \mu_{22} \ldots \mu_{2 n} \\
\vdots \vdots \mu_{j k} \vdots \\
\mu_{c 1} \mu_{c 2} \ldots \mu_{c n}
\end{array}\right\} .
$$

In equation (2), $\mu_{j k}(1 \leq j \leq c, 1 \leq k \leq n)$ represents the membership degree of sample $x_{k}$ to the class $j$. At present, the most widely used FCA is Fuzzy C-means (FCM), which mainly obtains the membership degree of each sample point to all class centers by optimizing the objective function, to determine the class of sample points to achieve the purpose of automatic classification of sample data. Each sample is given a membership function belonging to each cluster, and the samples are classified by the membership value. Its objective function $J_{f}$ is

$$
J_{f}=\sum_{j=1}^{c} \sum_{k=1}^{n_{j}} \mu_{j k}^{b}\left\|x_{k}^{(j)}-m_{j}\right\|^{2} .
$$

In equation (3), $b$ refers to a weighted index, $b>1$, which is used to describe the fuzzy degree of clustering results. The best empirical values are adopted, of which $b=2$. Parameter $x_{k}$ stands for sample $k$ in the $X, m_{j}$ refers to the cluster center, and $\mu_{j k}$ is a membership function.

To make $J_{f}$ minimum, equations (1)-(3) are transformed into equation (4) to solve the problem of constraint optimization.

$$
\left\{\min \sum_{j=1}^{c} \sum_{k=1}^{n_{j}} \mu_{j k}^{b}\left\|x_{k}^{(j)}-m_{j}\right\|^{2}, \text { s.t. } \sum_{j=1}^{c} \mu_{j k}=1 .\right.
$$

In equation (4), $b$ represents a weighted index, $x_{k}$ stands for the sample $k$ in $X, m_{j}$ is the cluster center, and $\mu_{j k}$ refers 
to a membership function. The Lagrange multiplier method is used to solve the problem as

$$
L=\sum_{j=1}^{c} \sum_{k=1}^{n} \mu_{j k}^{b}\left\|x_{k}-m_{j}\right\|^{2}-\sum_{k=1}^{c} \lambda_{k}\left[\sum_{j=1}^{c} \mu_{j k}-1\right] .
$$

In equation (5), $b$ refers to a weighted index, $x_{k}$ stands for sample $k$ in $X, m_{j}$ represents the cluster center, and $\mu_{j k}$ is a membership function , $L$ denotes the Lagrange multiplier method, and $\lambda_{k}$ refers to a multiplier of the function. Then, the partial derivatives of $L$ about $m_{j}, \mu_{j k}, \lambda_{k}$ are set to zero as follows:

$$
\mu_{j k}=\frac{\left\|x_{k}-m_{j}\right\|^{2 / 1-b}}{\sum_{l=1}^{c}\left\|x_{k}-m_{l}\right\|^{2 / 1-b}} .
$$

In equation (6), $\mu_{j k}$ represents a membership function, $b$ refers to a weighted index, $x_{k}$ stands for sample $k$ in $X$, and $m_{j}$ represents the cluster center.

$$
m_{j}=\frac{\sum_{k=1}^{n} \mu_{j k}^{b} x_{k}}{\sum_{k=1}^{n} \mu_{j k}^{b}} .
$$

In equation (7), $m_{j}$ is the cluster center, $b$ refers to a weighted index, $x_{k}$ stands for sample $k$ in $X$, and $\mu_{j k}$ represents a membership function. Figure 1 demonstrates the concrete steps of FCM.

First, parameter $c$ is set as the number of categories of fuzzy clustering, $b$ as a weighted index, $I$ as the number of iterative operations, and $\varepsilon$ as the threshold to stop iteration. Second, the clustering center is initialized. Third, iterative operation is carried out, the membership function is updated by equation (6), and the clustering center is updated by equation (7) until the result converges, so that the final result of clustering center and fuzzy partition matrix can be obtained [8].

Applying the fuzzy clustering technology to the educational management system, valuable information and its inherent laws can be excavated from the large amount of data in the educational management system. It helps improve the efficiency of school enrolment and employment management, teacher personnel management, educational management, student growth guidance and management, and asset and equipment management, to better understand students, optimize teaching, strengthen management, and improve relevant systems.

$\mathrm{CF}$ algorithm is a technology in information filtering and information system. It is used to analyze the users' interest, find the similar user of the specified user in the user group according to his or her interest, synthesize the evaluation of these similar users on a certain information, and form a system to predict the preference of the specified user for this information. The prediction of students' performance can be understood as the "recommendation" of students' performance, and the more similar the situation or factors between students, the more closer the students' examination scores. Predicting a student's performance can use the historical student data to find similar students to be predicted.

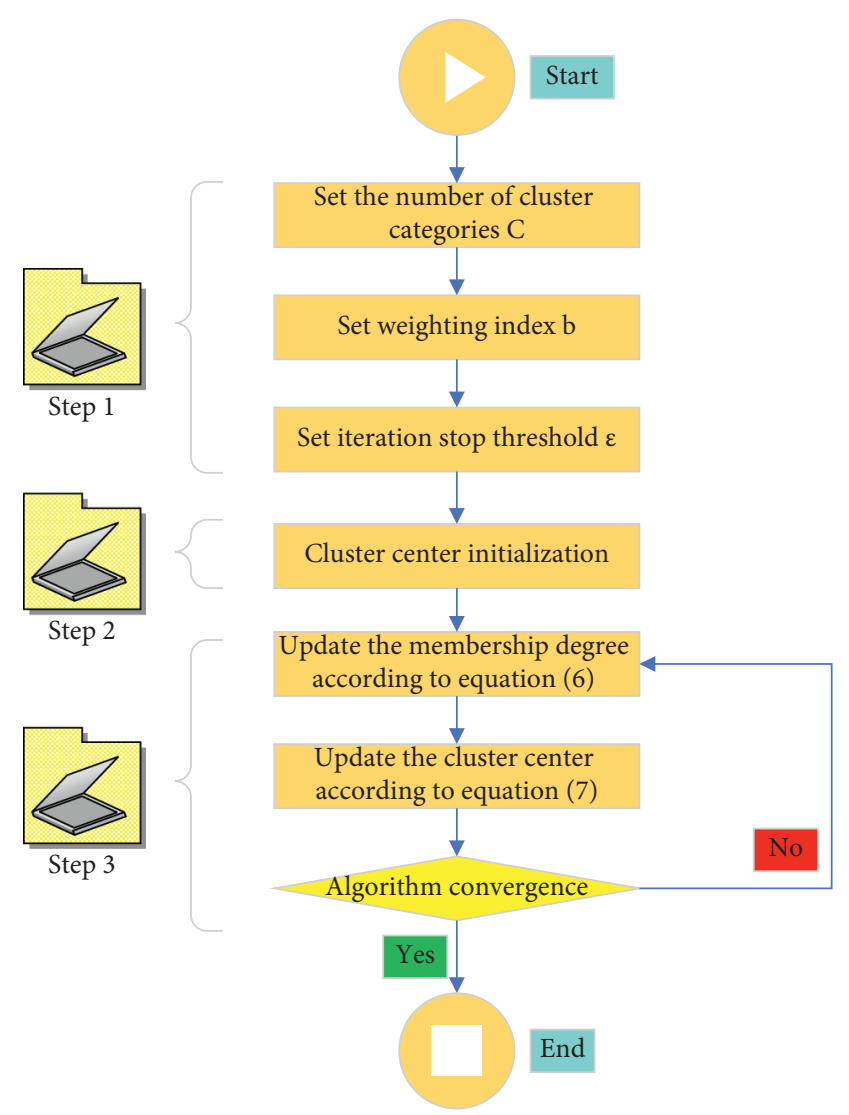

FIgURE 1: The concrete steps of FCM.

Students correspond to similar users in the collaborative filtering data set. These similar students' historical results can be integrated to predict the performance of the designated students. The present work combines FCM-CF to propose a student achievement prediction model based on FCM-CF. Figure 2 displays the structure of the model.

Initially, the fuzzy clustering technology is used to cluster the historical students' achievement data, and the membership matrix of each student in each cluster is obtained. Then, the prediction of each cluster to predict students' performance is calculated, according to the distribution of each student belonging to each cluster and the collaborative filtering method based on students. Eventually, the final prediction results are obtained by weighting the distribution of the target students belonging to each cluster. Specific prediction algorithms are as follows:

Through subordinate matrix- $U$ and the situation of each student belonging to each clustering center, the performance of student- $s$ ' in cluster- $c$ can be calculated [9] as

$$
\operatorname{per}\left(C_{j}\right)=\frac{\sum_{i=0}^{m-1} u_{i j} P_{i n-1}}{\sum_{i=0}^{m-1} u_{i j}} .
$$

In equation (8), $u_{i j}$ refers to the element in $U, P_{i n-1}$ represents the performance of student $s^{\prime}$ under the $n-1$ influencing factors, $m$ denotes the number of students, and $\operatorname{per}\left(C_{j}\right)$ means performance of student- $s^{\prime}$ in cluster- $c$. 




Figure 2: Structure of FCM-CF model.

Performance of $s^{\prime}$ under the factor $n$ can be obtained according to which cluster the students is subordinate.

$$
\operatorname{FCMCF}\left(s^{\prime}, n\right)=\sum_{j=0}^{c-1} u_{m j} \operatorname{per}\left(C_{j}\right) .
$$

In equation (9), $u_{m j}$ refers to an element in $U$, and $\operatorname{FCMCF}\left(s^{\prime}, n\right)$ stands for performance of the target student$s^{\prime}$ under the factor $n$.

2.2. Analysis of System Design. The development of technologies and the continuous advancement of informatization tremendously influence people's life, including the convenience brought by information technology and the troubles brought by information leakage. To keep up with the pace of the time, the requirements for modernization of all walks of life are increasing day by day. Informatization does not completely represent modernization. However, the realization of modernization must be inseparable from the development of informatization [10]. For modern college education, information technology is an indispensable method and a common used tool in the trend of educational reform. With the application of computer and other information technologies, teaching and learning in higher education have broken through the limitations of traditional teaching mode and become more flexible and controllable. With the advancement of educational informatization, in the continuous development, colleges inevitably face the situation that massive educational information needs to be processed. Although the information management systems of colleges are constantly improved, the current educational administration management system is still facing enormous challenges [11]. Using FCM to manage the educational information can provide data support for colleges to improve teaching quality. Additionally, the powerful data mining ability of FCM can also make colleges better handle the real-time situation of education, thus providing theoretical basis for the management decision-making of colleges [12].

The original intention of the educational administration management system is to manage the teaching work in colleges, and its functions need to meet the daily operation needs of colleges [13]. The requirements of the functions of education management system mainly include:

(1) Construction and management of academic status database, and input and editing of academic information.

(2) Formulating overall education plan for the institute, releasing teaching syllabus for the institute, and making teaching plan for teachers and reviewing on teaching plan from academic administration.

(3) Input, inquiry, editing, and statistic of the academic materials.

(4) Releasing information of curriculum from academic administration, inquiry, and selecting courses by students, integrating the information of courses selection, and reselecting courses.

(5) Arrangement of location and time of examination, selection and determination of the invigilator, and other inquiry of related information.

(6) Input and editing of the students' scores by teachers, statistical analysis on the students' personal performance by system automatically, and inquiry and complaints from students.

(7) Accomplishing self-evaluating of teachers, evaluation from students, colleagues, and leaders, and statistic analysis of the evaluation information. 
The design of the system is aimed at improving the educational management level of colleges, which makes the educational management work systematic, standardized, and intelligent, and reducing the manpower consumption required for information management in colleges. As a conclusion, the design of educational administration management system has five basic principles [14] as displayed in Figure 3.

(1) The principle of management institutionalization: The design of educational administration management system must be consistent with the educational administration management system of colleges. And the corresponding educational standards and systems should be taken as the benchmark to ensure the practicability of the system.

(2) The principle of openness and sharing of the system: To share information and data and facilitate users to operate across platforms, the design of the system should be kept open.

(3) The principle of simple and quick operation: Most of the time, the management of educational administration is relatively trivial. Therefore, the operation of the system must be simple and quick to facilitate the management of managers.

(4) The principle of convenient updating and upgrading: The complexity and diversity of information data require that the educational administration management system be convenient for updating, upgrading, and facilitating the subsequent improvement of the system.

(5) The principle of safety and reliability: As a tool of educational management in colleges, the educational management system must have a high degree of safety and stability. On the premise of ensuring that confidential information will not be leaked, the system cannot easily collapse with the operation.

\subsection{Design of Educational Administration Management System}

2.3.1. Design of Hardware of the System. The educational management system of college is composed of server, client, and network equipment. The system constructed here has two servers, namely, database server and network server. The former provides users with data query and instruction submission services, and the latter provides network connection services [15]. The instructions submitted by users are transmitted to the server through the network server, switches, firewalls, and route routers. After the server processes the instructions, the information is fed back to the user client [16]. Figure 4 displays the specific hardware architecture of the system.

2.3.2. Microsoft SQL Server 2008 Database. Microsoft SQL Server is a comprehensive database platform released by Microsoft, which has advantages such as great convenience and flexibility, and is very practical and highly integrated with other software [17]. Microsoft SQL Server 2008 is the most powerful and comprehensive version of Microsoft SQL Server, which has characteristics as following:

(1) Credibility: this characteristic makes application software of enterprise safe, reliable and expandable when carrying out important missions.

(2) High effectiveness: this characteristic reduces the time and cost when enterprise conduct developing and managing on infrastructure of data

(3) Intelligence: the development platform can provide related data for users, as a comprehensive and flexible platform [18]

Microsoft SQL Server 2008 has 4 kinds of core components as following:

(1) Database engine: mainly used to storage, manage, and control the access and other handling on the data

(2) Analysis service: providing supports for online analytical processing and data mining based on existing database

(3) Reporting service: presenting the generated classified information based on existing data in the reports, thus making users can access the reports and the data

(4) Integrating service: integrating data, processed results, and processing reports of the core component to realize the integration of the data

Figure 5 demonstrates the architecture of Microsoft SQL Server 2008.

2.3.3. Structure Design of the System. The Client-Server (C/ S) mode takes the server as the center, which can make full use of the hardware advantages of the server and the client, allocate tasks reasonably, and reduce the communication time of the system. The client can access the server to obtain the required network resources, and the information uploaded by the client can be stored on the server. Figure 6 manifests the structure of $\mathrm{C} / \mathrm{S}$ system.

Browser-Server mode $(\mathrm{B} / \mathrm{S})$ is an improvement of $\mathrm{C} / \mathrm{S}$, which belongs to three-layer $\mathrm{C} / \mathrm{S}$ structure. $\mathrm{B} / \mathrm{S}$ structure only needs a browser to fix the problem that the traditional way must use special software to solve, which greatly saves costs and simplifies the development, maintenance, and use of the system. It is a new software system construction technology. This model unifies the client and concentrates the core part of the system function realization on the server. Data interaction can be achieved by installing a browser on the client and a database on the web server [19]. Figure 7 signifies the structure of $\mathrm{B} / \mathrm{S}$ system.

The system or software using B/S structure can have the server to complete the installation, modification, and maintenance functions. The biggest advantage of $\mathrm{B} / \mathrm{S}$ structure is that whenever and wherever users want a computer that can connect to the Internet, they can achieve the purpose of zero maintenance of the client without installing special software. Both structures have their own 




FIgURE 3: Design principles of educational administration management system.

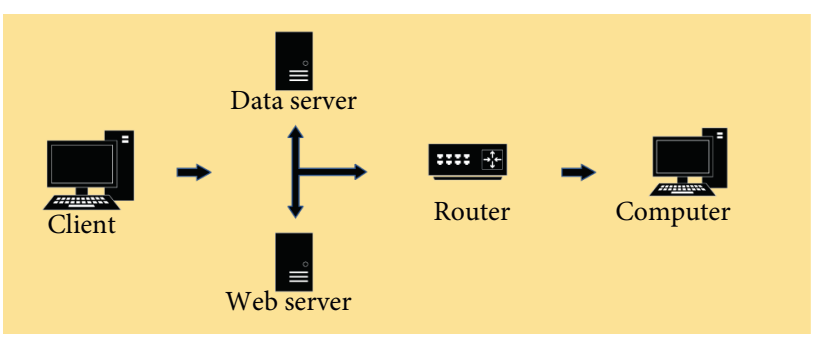

FIGURE 4: Hardware architecture of educational management system.

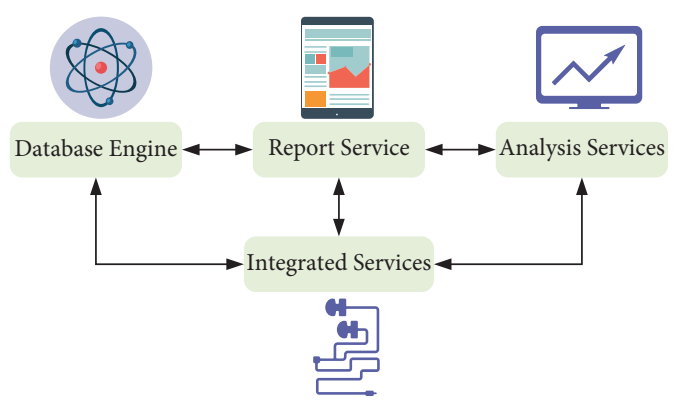

Figure 5: Architecture of Microsoft SQL Server 2008.

advantages and disadvantages [20], as shown in Figures 8 and 9.

In view of the advantages and disadvantages of $\mathrm{B} / \mathrm{S}$ structure and $\mathrm{C} / \mathrm{S}$ structure, the present work mainly discusses the integration of the two architectures. At present, there are two ways for $\mathrm{C} / \mathrm{S}$ and $\mathrm{B} / \mathrm{S}$ mixed model: one is

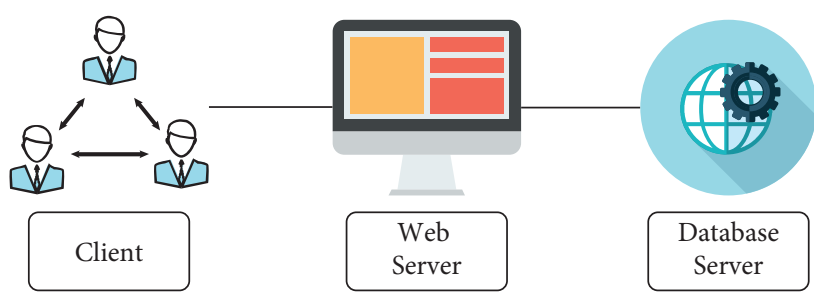

FIGURE 6: Structure of C/S system.

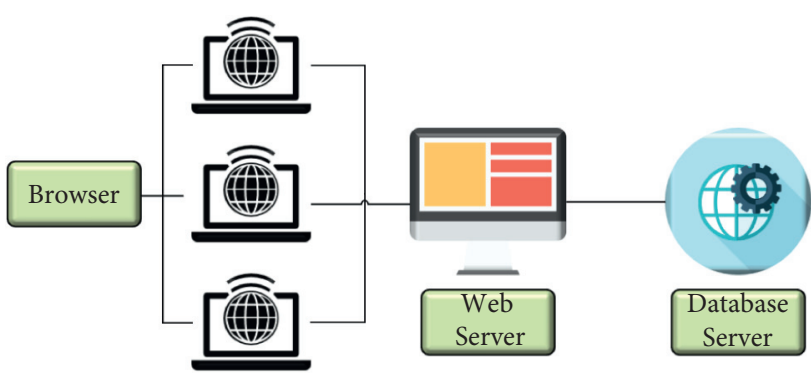

Figure 7: Structure of B/S system

called "inquiry/modifying" model, and the other is called "inside/outside" model.

In the $\mathrm{B} / \mathrm{S}$ and $\mathrm{C} / \mathrm{S}$ mixed software architecture of the "Inquiry/modifying" model, regardless of the way users are connected to the system via Internet or LAN, the C/S architecture is used if maintenance and modification of data operations are required. If only general query and browsing operations are performed, the $\mathrm{B} / \mathrm{S}$ architecture is used. 


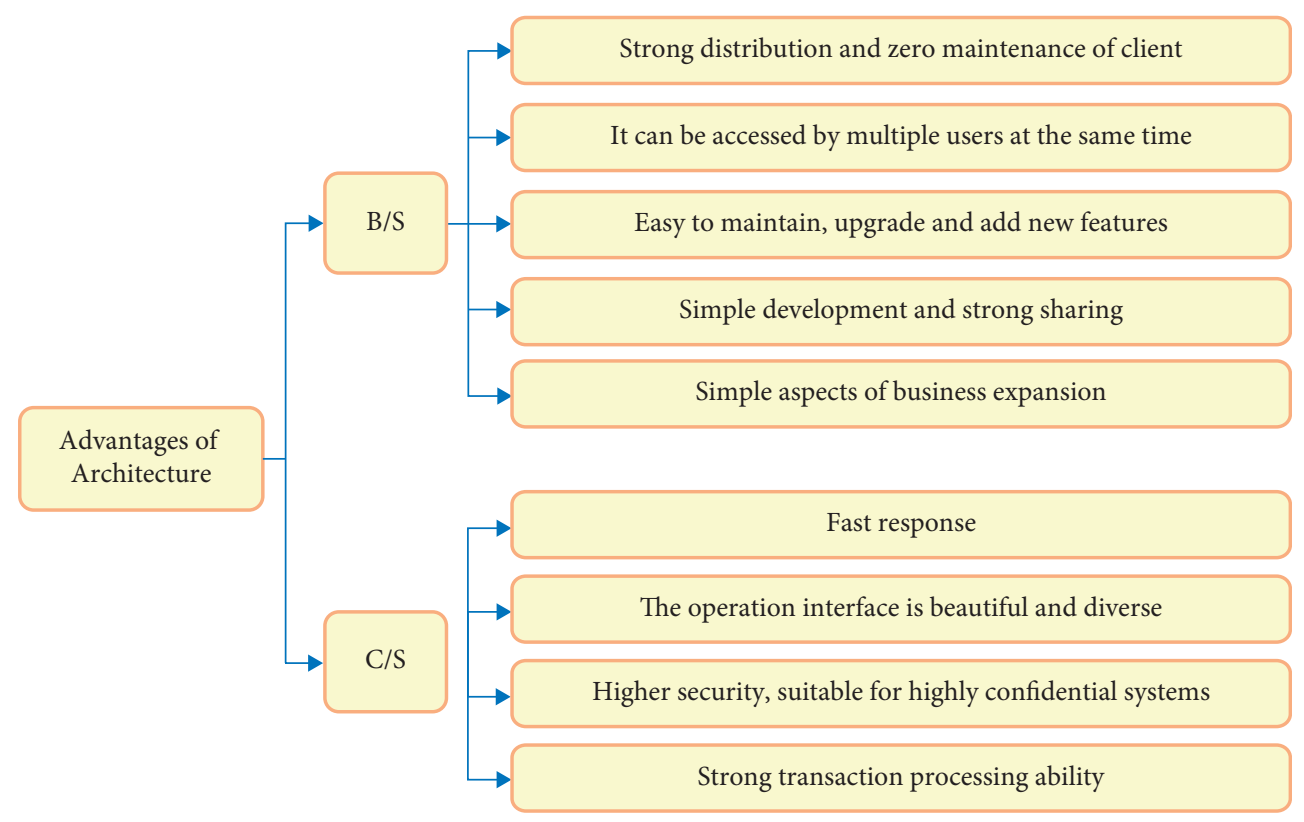

FIGURE 8: Comparison between advantages of $\mathrm{B} / \mathrm{S}$ structure and $\mathrm{C} / \mathrm{S}$ structure.

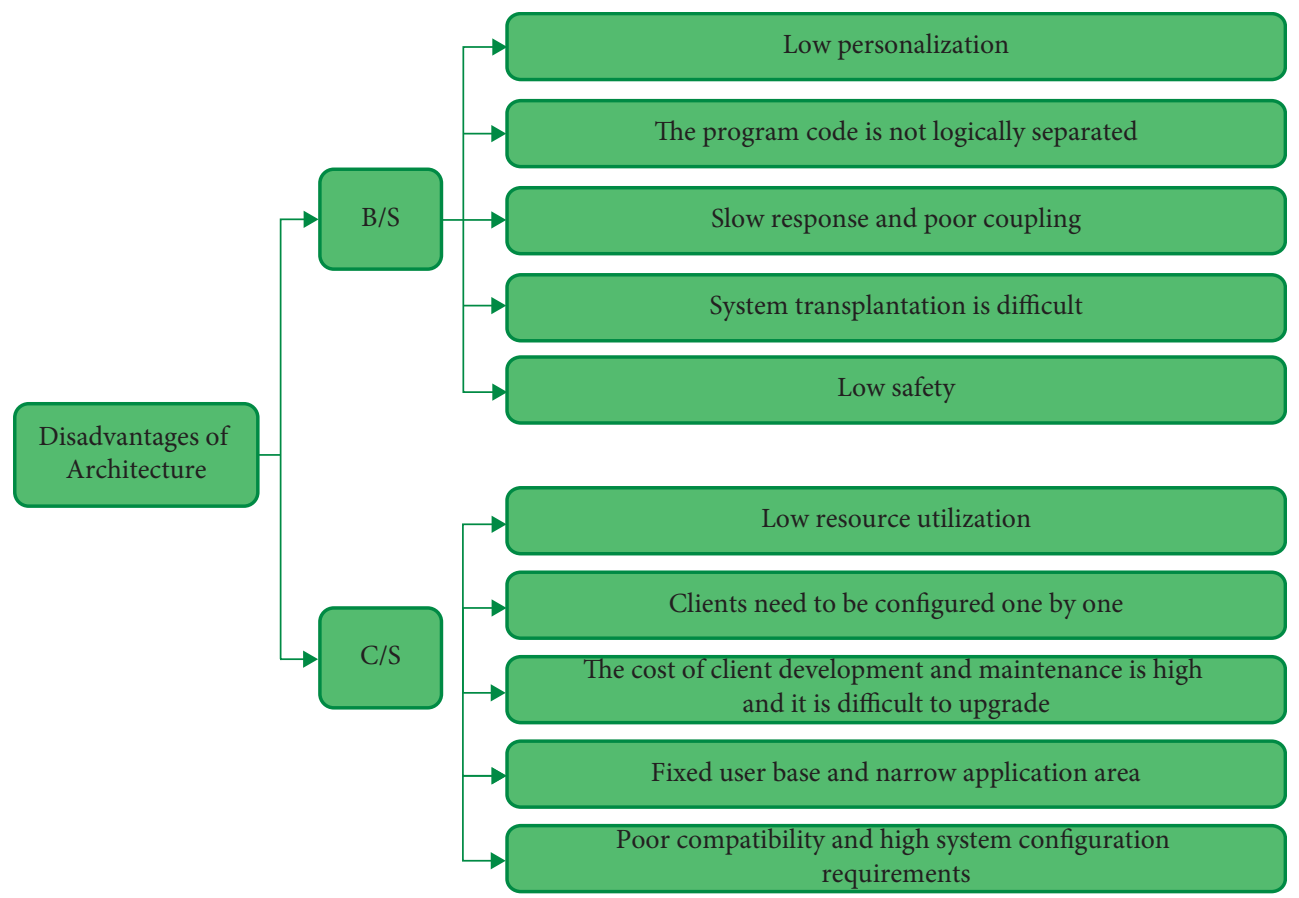

FIGURE 9: Comparison between disadvantages of $\mathrm{B} / \mathrm{S}$ structure and $\mathrm{C} / \mathrm{S}$ structure.

In the "inside/outside" model of $\mathrm{B} / \mathrm{S}$ and $\mathrm{C} / \mathrm{S}$ mixed software architecture, the outside users of enterprise access network server through Internet and then access database server through network server. The internal users of the enterprise directly access the database server through the LAN, and the software system adopts the C/S architecture.

The software architecture of the educational administration management system constructed is combined with Browser $\backslash$ Server $(B \backslash S)$ and Client $\mid$ Server $(C \backslash S)$, and the internal LAN is used to store, manage, and maintain the educational administration information [21]. Different roles correspond to different management paths. Students and teachers need functions such as inquiring and entering educational information, while the Academic Affairs Office needs functions such as reviewing and maintaining educational information [22]. Figure 10 signifies the specific software architecture.

Based on the functional requirements of the system mentioned in the previous section, the seven main 


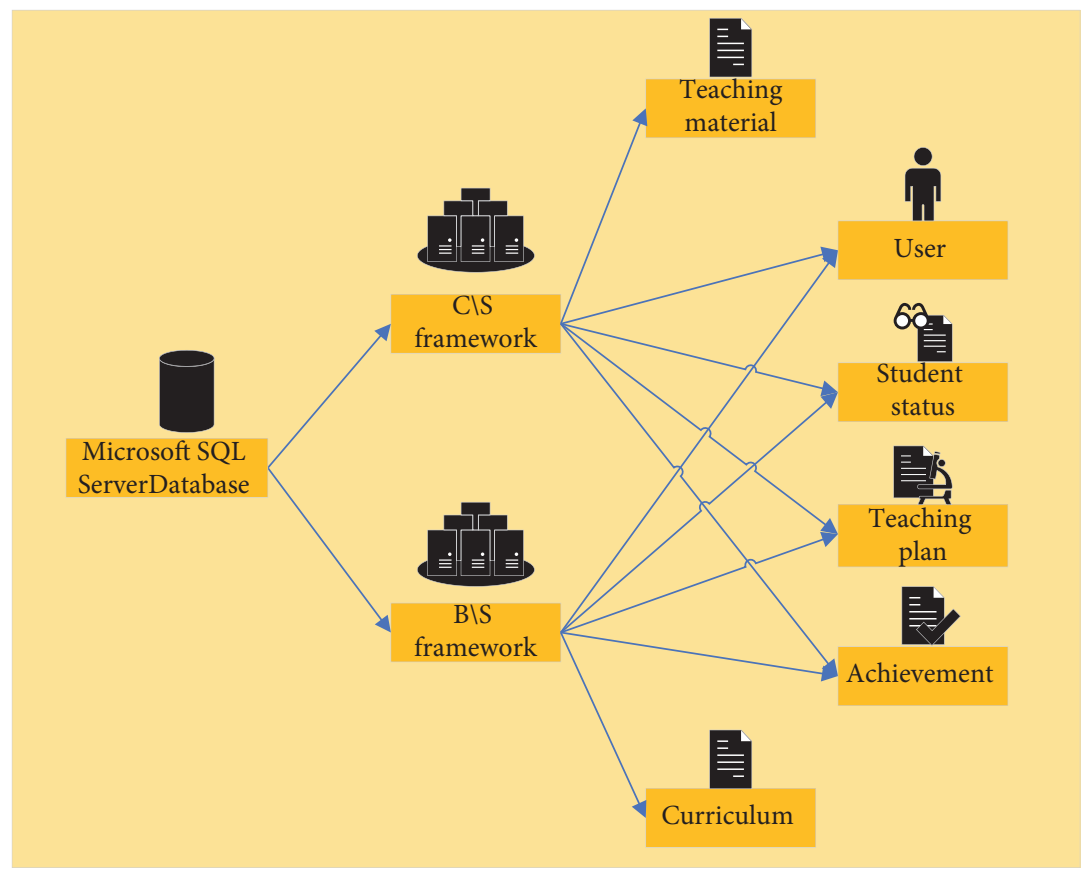

Figure 10: Software architecture of educational administration system.

functions are designed for the educational administration management system, which are student status information, annual teaching plan, teaching materials, online course selection for students, ability test for each semester, statistical entry and query of test results, and evaluation management for teachers [23]. Figure 11 illustrates the specific operation flow.

Figure 12 demonstrates the main functions of the 7 modules of educational management system.

Academic status information management includes three parts: information management, daily management, and affairs management. The management of the teaching plan mainly refers to the fact that the academic administration formulates and publishes the overall teaching plan of the academic year through the system, and the teachers formulate and upload the teaching plan according to the syllabus and teaching tasks. The teaching material management module is used for collecting of teaching material demand information and the input of teaching material purchase and distribution records; students' online course selection management mainly provides the selection and confirmation functions of compulsory courses and elective courses; semester test management is mainly used for inquiry, affirming, arranging, and releasing examination time, examination room information, and invigilator information; the performance management module provides the functions of input, query, and complaints of test scores. Present work applies the FCM-CF algorithm to this module, which can realize the prediction of students' future scores based on students' historical scores. Teachers' evaluation and management module is mainly used to input and edit information related to teachers' evaluation.

\subsection{System Simulation Experiment Design}

2.4.1. Selection of Dataset and Transition of Data. The present work mainly takes student achievement prediction as an example to carry out the performance test research of prediction model. Students' academic performance is related to many factors. Extracurricular learning is as important as inclass learning. Students' personal interest in learning, learning ability, and learning methods directly affect students' examination scores. The investigation of students' extracurricular learning provides a more comprehensive grasp of the factors affecting students' performance. The present work takes the survey results collected by the " $\mathrm{C}$ language programming" course of Computer Science and Application specialty and students' examination scores of the course as an example and selects the fields that have a greater impact on academic performance, such as preclass preparation, classroom performance, attendance rate, extracurricular learning, and homework completion of students, to predict their examination scores of the course. Table 1 lists the specific data.

For the convenience of statistics, the preclass preparation, classroom performance, attendance, extracurricular study, and homework completion in Table 1 are converted into corresponding scores $(0 \sim 10)$. The students' exam scores are divided by 10 , for example, 60 points into 6 .

A dataset contains all the students' scores. The dataset is divided into training set and test set to verify the effect of each prediction method.

2.4.2. Specific Design of Experiment Flow. According to the educational management system constructed above, FCM-CF model is used to predict students' exam scores. 


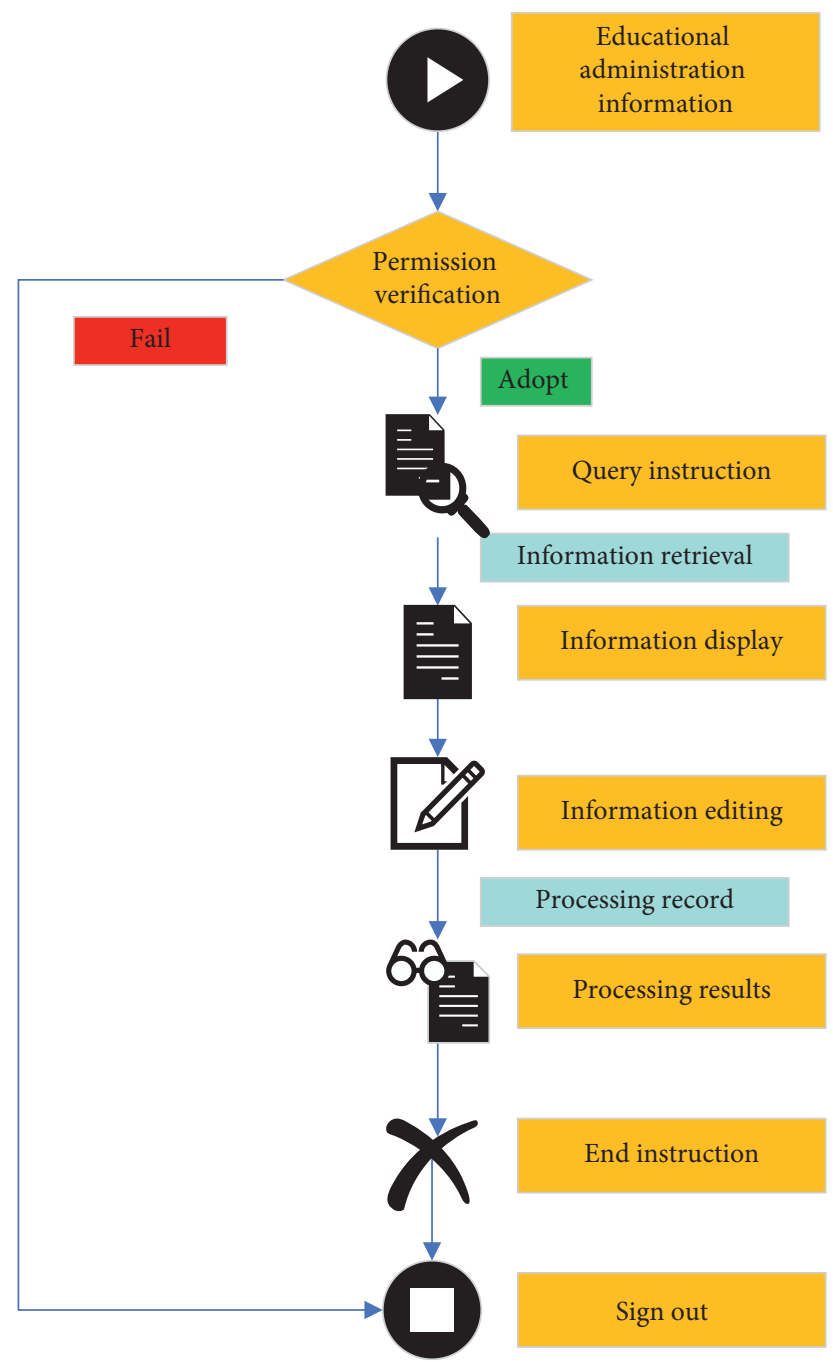

FIgURE 11: Operation flow of educational administration information management.

To verify the validity of the model, $K$-Means clustering ( $K$-Means) is selected to replace the FCM-CF model to form a comparison. The historical performance data set of students is used to predict the performance of students, and the prediction results are compared and analyzed. When the two algorithms are used for prediction, the number of cluster (parameter $c$ ) needs to be determined first. In this process, the data set is temporarily divided into training set and test set in a ratio of $9: 1$. Simultaneously, it is necessary to determine the number $K$ of the nearest neighbor in $\mathrm{KNN}$ algorithm. In the experiment, the $K$ value is initially taken as $10 \%$ of the size of the training set and increased at an interval of $5 \%$.

In the FCM-CF prediction model, the whole training set refers to the students whose exam scores are to be predicted, and data of all students are divided into different clusters according to different probabilities of the prediction results. Accordingly, different weights are used to predict the students' scores. Hence, the present work explores the best proportion of training set to data set when using FCM-CF prediction model. Figure 13 shows the specific process.
2.4.3. Evaluation Indexes. The mean absolute error (MAE) is used as the standard of prediction and evaluation [24]. The smaller the value of MAE, the higher the prediction accuracy. Equation (10) shows the calculation.

$$
\text { MAE }=\frac{\sum_{i=1}^{N}\left|\widehat{r}_{u i}-r_{u i}\right|}{N} .
$$

In equation (10), $\widehat{r}_{u i}$ refers to prediction score of user- $u$ to project- $i$, namely, the prediction score of the target students' test scores by the prediction method. Parameter $r_{u i}$ stands for actual score of user- $u$ to project $-i$, namely, the actual test scores of the target students. $\mathrm{N}$ represents the number of scores, which user- $u$ makes on the project $-i 3$.

\section{Results of Simulation Experiment}

3.1. Display of Forecast Data. Figure 14 demonstrates the transition results of students' exam scores.

Figure 14 shows that the students' final achievement is closely related to their daily study performance. Whether to prepare before class, listen carefully in class, attend every 


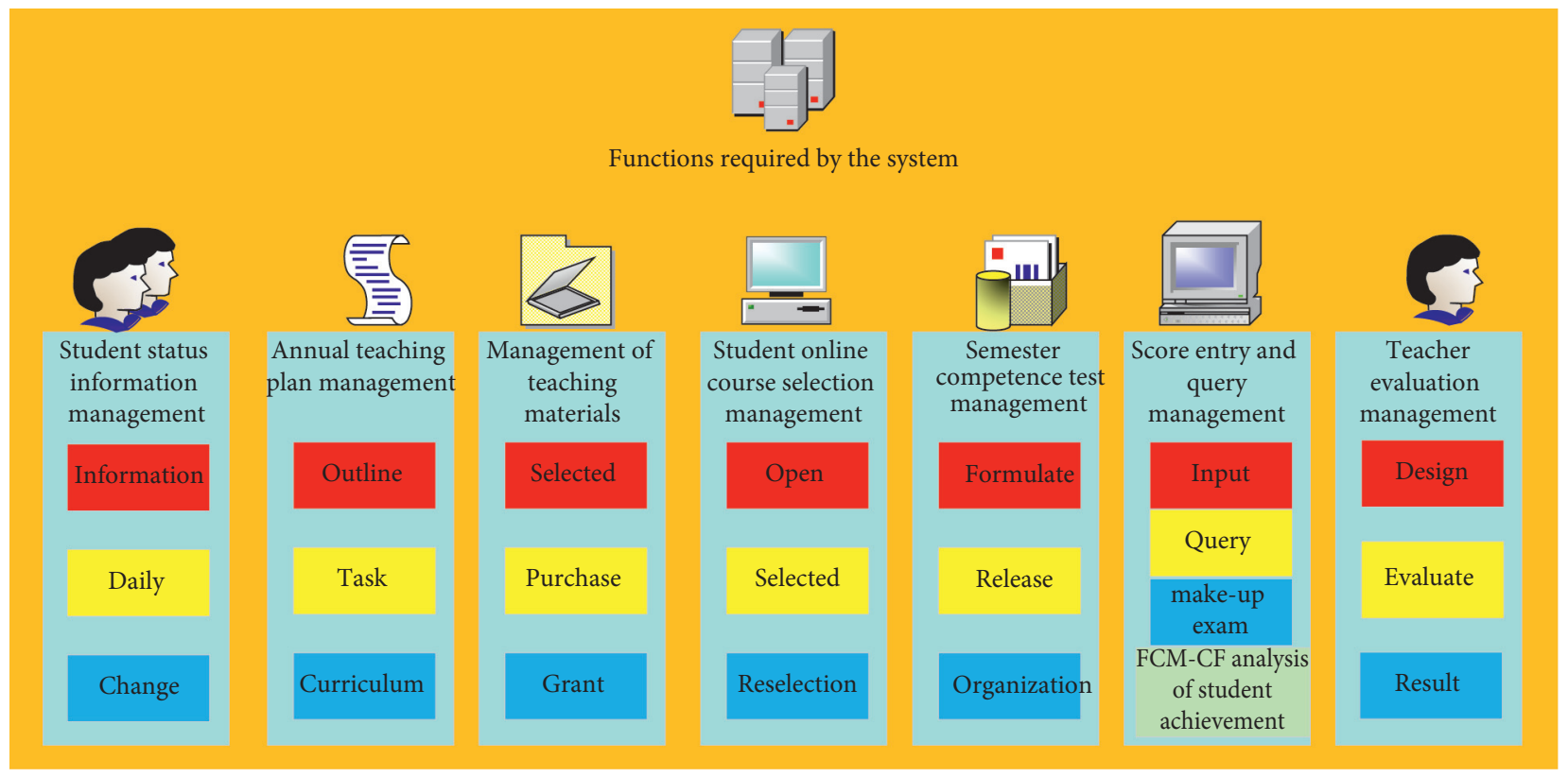

FIGURE 12: Main functions of each module in the educational management system.

TABLE 1: Students' performance prediction results.

\begin{tabular}{|c|c|c|c|c|c|c|}
\hline Student number & Preview before class & Classroom performance & Attendance & Out-of-class activities & Operation & Exam score \\
\hline 1 & Basically not & Very bad & Poor & Basically not & Very bad & 27 \\
\hline 2 & Complete preview & Excellent & Good & Occasionally & Excellent & 90 \\
\hline 3 & Basic preview & Commonly & Good & Occasionally & Secondary & 68 \\
\hline 4 & Good preview & Good & Excellent & Often & Good & 81 \\
\hline 5 & Partial preview & Commonly & Commonly & Very seldom & Secondary & 86 \\
\hline 6 & Basic preview & Poor & Excellent & Often & Poor & 59 \\
\hline$\ldots$ & $\ldots$ & $\ldots$ & $\ldots$ & $\ldots$ & $\ldots$ & $\ldots$ \\
\hline
\end{tabular}

class, carry out related study after class, complete homework, etc. will directly affect the students' final achievement. Under normal circumstances, students' scores corresponding to these performances will be similar; that is, students who have prepared well before class will perform very well in class, their attendance rate will be relatively high, and their extracurricular study time will be longer. Meanwhile, the completion of their homework is also very good, and correspondingly, their exam scores will be satisfactory. On the contrary, students who do not preview before class will perform poorly in class, and their attendance rate will be relatively low. They will not study after class, the quality of their homework will be poor, and their final exam results will be unsatisfactory.

\subsection{Determination of the Indexes of KMCF Model and FCM-CF Model}

3.2.1. Determination of the Indexes of the Model. Figure 15 reveals the variation trend of the predicted mean absolute error (MAE) of the KMCF model as the number of clusters $c$ in the $K$-means clustering changes from 1 to 25 , and $K$ takes $10 \%$ to $40 \%$ of the training set.

Figure 15 reveals that it is the optimal, when the number of clusters is 10 , and the $K$ value is $20 \%$ of the training set.
When the number of clusters is 1 , that is, when all students are in the same cluster, the $K$-means clustering is not taken to cluster students. As the number of clusters increases, and the number of clusters is relatively small, more students in the same cluster with the students whose scores are to be predicted will be given higher authority, and students being in the same cluster can be considered to have higher similarity. However, when the number of clusters is too large, even when each student is a cluster, clustering should not be taken under this situation.

With the increase of the nearest neighbor size $K$, more students similar to the students to be predicted are divided into the nearest neighbor set, which will improve the accuracy of prediction. However, the $K$ value continues to increase to be even equivalent to that of the entire training set, and students who are very different from the students to be predicted are also divided into the $\mathrm{KN}$ set, thus reducing the accuracy of KMCF prediction method. When the FCMCF model is used for prediction, the number of clusters $c$ in the clustering is kept as 10 of the KMCF model.

3.2.2. Determination of Training Set Ratio of FCM-CF Model. Figure 16 illustrates the variation trend of MAE along with the change of training set ratio. 


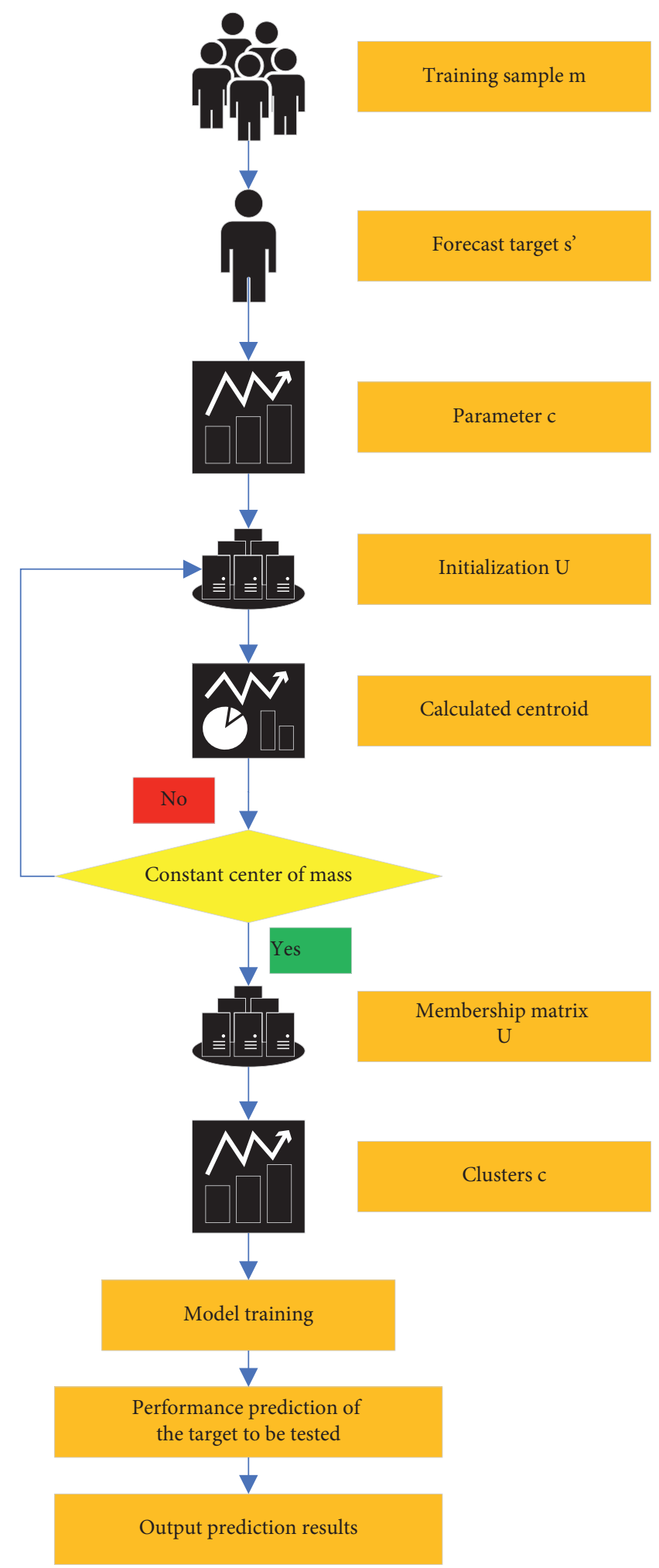

FIGURE 13: Clustering of students' scores and flow of predictions.

Figure 16 indicates that when the FCM-CF is adopted to carry out prediction, the prediction error of the model increases with the increase of the proportion of the training set. When the proportion of the training set is $90 \%$, the error of the model reaches the minimum. This is because when the size of the training set increases, more data of students' performance will be involved in the prediction, avoiding some accidental factors, so that the smaller the MAE value of the test set, the better the prediction effect. Therefore, the proportion of training set in data set is set to $90 \%$. 


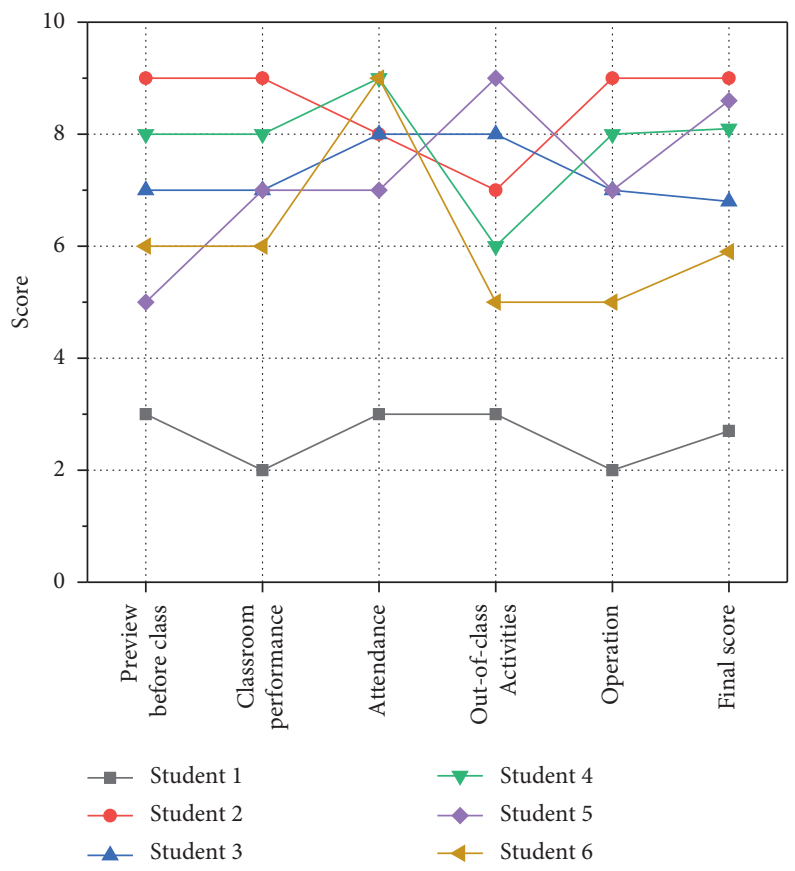

FIgURE 14: Students' scores prediction curve.

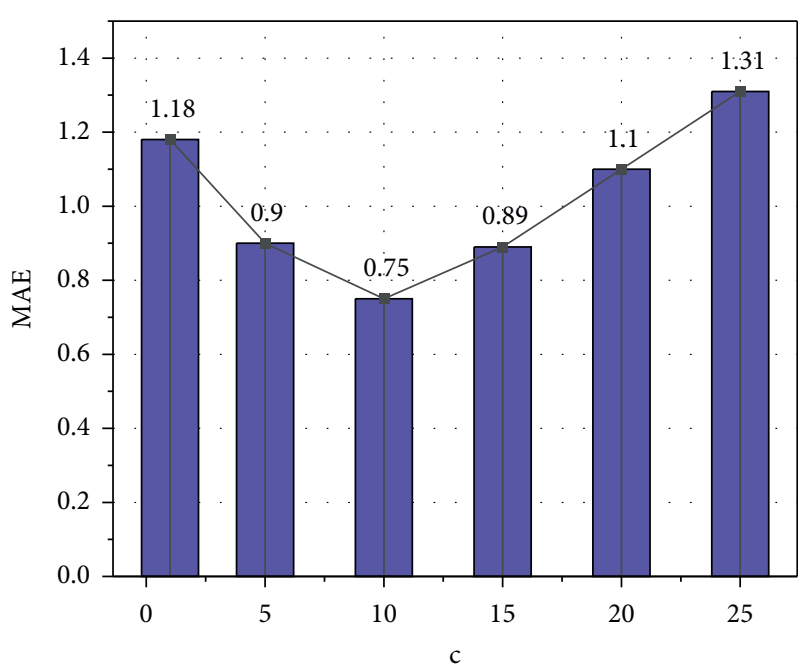

(a)



(b)

FIGURE 15: Clustering of students' scores and flow of predictions. (a) Variation trend of MAE with the change of the number of cluster $c$; (b) variation of MAE with the change of closest neighbor $K$.

3.3. Comparative Analysis of Prediction Performance of Models. To get a clearer comparison between KMCF and FCM-CF models, comparisons are made on the MAE results predicted by the two models under different training set ratio. Figure 17 denotes the comparison results.
Figure 17 implies that the prediction MAE of KMCF and FCM-CF models declines with the growth of the proportion of training set to data set, and the models have the lowest prediction MAE when the proportion of training set is $90 \%$. The average MAE predicted by KMCF model is 0.939 . The average MAE of FCM-CF model is 0.838 , lower than that of 


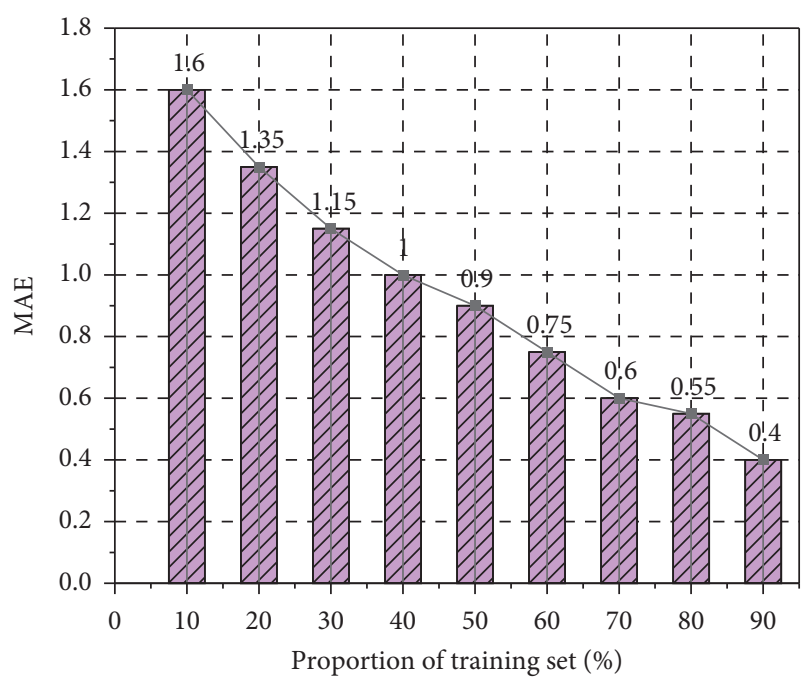

FIGURE 16: Variation trend of MAE along with the change of training set ratio.

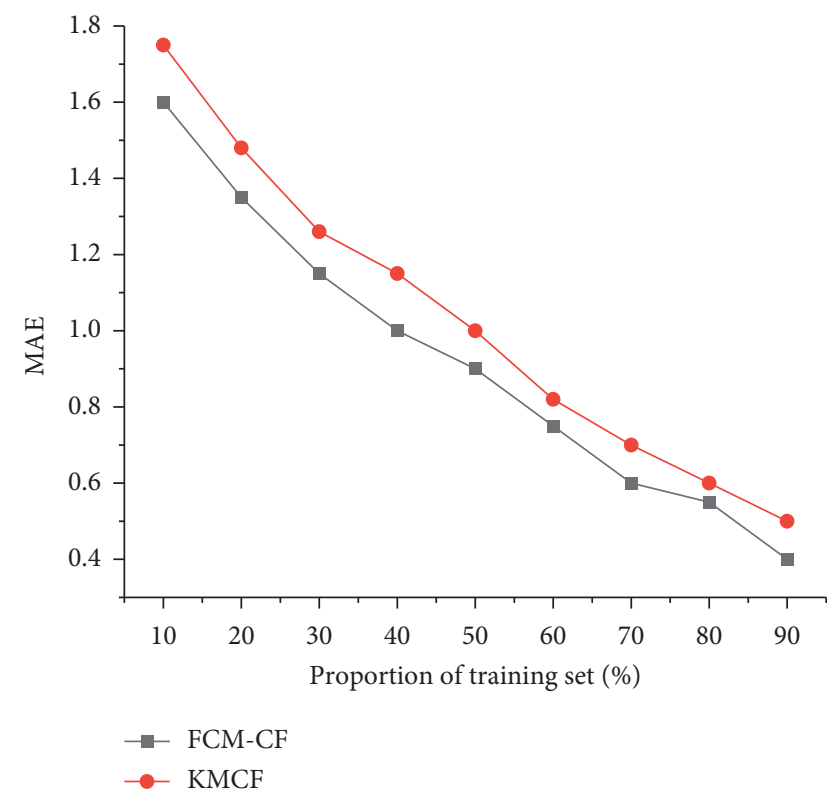

FIgURE 17: Variation trend of MAE along with the change of the training set size.

KMCF model $10.8 \%$. Therefore, under the same conditions, the prediction error of FCM-CF model is lower, which is more suitable for the prediction of middle school students' performance in educational management system.

\section{Conclusion}

With the progress of the times, colleges are inevitably faced with the situation that massive educational information needs to be processed in the continuous development, and the educational administration of colleges is in urgent need of being made further intelligent. The present work proposes a student achievement prediction model based on FCM-CF, introduces it into the research of educational management, and constructs an intelligent educational management system. Initially, the FCM-CF model is described in detail. Next, the system requirements and specific design methods are expounded. Eventually, taking the student performance prediction as an example, the performance of the system is tested by designed simulation experiments. The results show that students' exam scores are closely related to their daily learning performance. Under the same experimental conditions, the prediction error of FCM-CF model proposed here is less than $10.8 \%$ of other algorithms, which has better prediction performance and is more suitable for the prediction of middle school students' scores in educational administration management system. The innovation is to introduce FCM-CF algorithm into the educational administration system model, giving it the function of data mining and intelligent performance prediction. There are some shortcomings, such as incomplete coverage of the factors affecting students' grades. Additionally, there is room for optimization of the operation efficiency of the algorithm provided. Subsequent research will solve the above problems. The research purpose is to provide important technical support for the educational management of colleges to be more intelligent and reduce the waste of manpower.

\section{Data Availability}

The labeled dataset used to support the findings of this study is available from the corresponding author upon request.

\section{Conflicts of Interest}

The author declares no competing interests.

\section{References}

[1] R. Shang, C. Chen, G. Wang, L. Jiao, M. A. Okoth, and R. Stolkin, "A thumbnail-based hierarchical fuzzy clustering algorithm for SAR image segmentation," Signal Processing, vol. 171, Article ID 107518, 2020.

[2] D. Mújica-Vargas, "Superpixels extraction by an intuitionistic fuzzy clustering algorithm," Journal of Applied Research and Technology, vol. 19, no. 2, p. 141, 2021.

[3] S. Nasir, N. Nurhidayah, and A. Suprianto, "Effect of selfregulated learning on academic achievement of Puangrimaggalatung university educational administration study program students," International Journal of Educational Management and Innovation, vol. 1, no. 3, p. 205, 2020.

[4] Z. Lv, D. Chen, R. Lou, and A. Alazab, "Artificial intelligence for securing industrial-based cyber-physical systems," Future Generation Computer Systems, vol. 117, pp. 291-298, 2021.

[5] Z. Ning, X. Hu, Z. Chen et al., "A cooperative quality-aware service access system for social internet of vehicles," IEEE Internet of Things Journal, vol. 5, no. 4, pp. 2506-2517, 2018.

[6] Z. Lv, D. Chen, and J. Li, "Novel system design and implementation for the smart city vertical market," IEEE Communications Magazine, vol. 59, no. 4, pp. 126-131, 2021.

[7] N. Mahata and J. K. Sing, "A novel fuzzy clustering algorithm by minimizing global and spatially constrained likelihoodbased local entropies for noisy 3D brain MR image segmentation," Applied Soft Computing, vol. 90, Article ID 106171, 2020. 
[8] J. Li, Y. Ning, Z. Yuan, and C. Yang, "Research on medical image segmentation based on fuzzy clustering algorithm," Matrix Science Pharma, vol. 3, no. 1, p. 9, 2019.

[9] I. Oplatka, "School leadership for refugees' education: social justice leadership for immigrant, migrants, and refugees," Journal of Educational Administration and History, vol. 53, no. 1, pp. 1-3, 2020.

[10] N. Ibrahim Fantoukh, M. Maher Ben Ismail, and O. Bchir, "Automatic determination of the number of clusters for semisupervised relational fuzzy clustering," International Journal of Fuzzy Logic and Intelligent Systems, vol. 20, no. 2, pp. 156-167, 2020.

[11] H. Xu, R. Hou, J. Fan, L. Zhou, and J. Liu, "The unordered time series fuzzy clustering algorithm based on the adaptive incremental learning," Journal of Intelligent and Fuzzy Systems, vol. 38, no. 1, pp. 1-9, 2020.

[12] X. Hu, J. Cheng, M. Zhou et al., "Emotion-aware cognitive system in multi-channel cognitive radio ad hoc networks," IEEE Communications Magazine, vol. 56, no. 4, pp. 180-187, 2018.

[13] Y. Zhang, Y. Zhang, and R. Zhang, "Text information classification method based on secondly fuzzy clustering algorithm," Journal of Intelligent and Fuzzy Systems, vol. 38, no. 1, pp. 1-12, 2020.

[14] N. Y. Pehlivan and I. B. Turksen, "A novel multiplicative fuzzy regression function with a multiplicative fuzzy clustering algorithm," Romanian Journal of Information Science and Technology, vol. 24, no. 1, pp. 79-98, 2021.

[15] D. Tan, W. Zhong, C. Jiang, X. Peng, and W. He, "High-order fuzzy clustering algorithm based on multikernel mean shift," Neurocomputing, vol. 385, no. 8, pp. 63-79, 2020.

[16] L. H. Wen, Z. H. Shi, and H. Y. Liu, "Research on risk assessment of natural disaster based on cloud fuzzy clustering algorithm in Taihang mountain," Journal of Intelligent and Fuzzy Systems, vol. 37, no. 4, pp. 1-9, 2019.

[17] Z. Hayat and T. R. Soomro, "Implementation of Microsoft SQL server using 'AlwaysOn' for high availability and disaster recovery without shared storage," International Journal of Experiential Learning and Case Studies, vol. 3, no. 1, pp. 9-17, 2018.

[18] B. C. Gabriel and L. G. Kabari, "Hybridized concurrency control technique for transaction processing in distributed database system," International Journal of Computer Science and Mobile Computing, vol. 9, no. 9, pp. 118-127, 2020.

[19] Y. Li, L. Chen, D. Yu, and R. Gao, "Research and developing of evaluation information system using B/S structure and SQL server technology," Journal of Physics: Conference Series, vol. 1952, no. 4, Article ID 042088, 2021.

[20] S. K. Singh and A. K. Tiwari, "Design and implementation of secure computer based examination system based on $\mathrm{B} / \mathrm{S}$ structure," International Journal of Applied Engineering Research, vol. 11, no. 1, pp. 312-318, 2016.

[21] F. Zhao, C. Li, H. Liu, and J. Fan, "A multi-objective interval valued fuzzy clustering algorithm with spatial information for noisy image segmentation," Journal of Intelligent and Fuzzy Systems, vol. 36, no. 6, pp. 5333-5344, 2019.

[22] Y. Yang, J. Yu, Y. Fu, and J. Hu, "Research on geological hazard risk assessment based on the cloud fuzzy clustering algorithm," Journal of Intelligent and Fuzzy Systems, vol. 37, no. 2017, pp. 1-8, 2019.

[23] D. Sivaganesan, "Optimized wireless sensor node multidimensional routing using fuzzy clustering and chaotic gravitational search algorithm," IRO Journal on Sustainable Wireless Systems, vol. 3, no. 1, pp. 40-48, 2021.
[24] H. Peng, B. Hu, Q. Shi et al., "Removal of ocular artifacts in EEG-an improved approach combining DWT and ANC for portable applications," IEEE Journal of Biomedical and Health Informatics, vol. 17, no. 3, pp. 600-607, 2013. 